Cite this: RSC Advances, 2013, 3, 1460

\title{
Enzymatic synthesis of antibody-human serum albumin conjugate for targeted drug delivery using tyrosinase from Agaricus bisporus
}

\author{
Alexandra Rollett, a Barbara Thallinger, anna Ohradanova-Repic, ${ }^{\text {a }}$ \\ Christian Machacek, ${ }^{b}$ Evelyn Walenta, ${ }^{c}$ Artur Cavaco-Paulo, ${ }^{d}$ Ruth Birner-

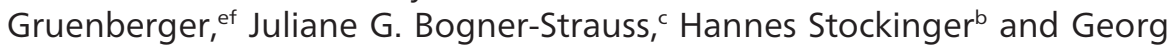 \\ M. Guebitz*af
}

\begin{abstract}
Highly specific targeted drug delivery devices can be obtained with antibody-human serum albumin (mAbHSA) conjugates. However, their conventional production involves several reaction steps including chemical modification and activation of both proteins followed by cross-linking often involving toxic chemicals. Here, we describe the enzymatic synthesis of mAb-HSA conjugates for targeted drug delivery devices using tyrosinase from Agaricus bisporus under mild reaction conditions $\left(\mathrm{pH} 6.8,25^{\circ} \mathrm{C}\right)$. Reaction conditions were optimized by using fluorescence labeled HSA to facilitate SDS-PAGE analysis with fluorescence scanning. Enzymatic cross-linking in the presence of natural low molecular weight phenolic compounds (e.g. caffeic acid) resulted in reaction products in the molecular weight range of $\sim 216 \mathrm{kDa}$, corresponding to mAb-HSA conjugates. The composition of the conjugates was confirmed with tryptic digestion followed by LC-MS/MS analysis of the resulting peptide fragments. Successful binding of mAbHSA conjugates (in contrast to free HSA) to MHC II molecules, located on antigen-presenting cells, was demonstrated by both ELISA and flow cytometry analysis.
\end{abstract}

Received 18th October 2012,

Accepted 21st November 2012

DOI: $10.1039 / \mathrm{c} 2 \mathrm{ra} 22560 \mathrm{c}$

www.rsc.org/advances
By using antibody-carrier-drug systems the specificity of antibodies is often exploited to achieve targeting to cancer cells. $^{4,5}$ HSA is widely used as a drug carrier ${ }^{6}$ while more recently we have described a strategy avoiding toxic chemicals for the production of HSA-nanoparticles. ${ }^{7}$ The use of mAb-HSA drug delivery devices based on this concept is described in the literature. ${ }^{8-10}$ The preparation of these mAb-HSA conjugates involves several reaction steps including the reduction of one reaction partner to give free thiol groups and the introduction of maleimide groups to the complementary protein prior to the actual coupling reaction. These activation steps are followed by time consuming gel filtration or dialysis. For the purification of the conjugates another filtration/washing step is needed.

In this study, we suggest the introduction of an enzyme in the production of mAb-HSA conjugates. Due to mild reaction conditions in terms of $\mathrm{pH}$-value, temperature and reaction environment (i.e. aqueous), enzymes are increasingly used for the replacement of sometimes even toxic chemicals. ${ }^{11-13}$

Tyrosinase, for example, was demonstrated to improve the texture of dough for bread production by oxidation of low molecular weight phenolic compounds of flour and by crosslinking of gluten proteins. ${ }^{14}$ Several research groups used tyrosinase to produce protein-polysaccharide conjugates to obtain biomaterials with special mechanical and chemical 
properties. ${ }^{15-17}$ In the biomedical field tyrosinase cross-linking was used to enhance the properties of biomaterials: the antioxidant activity of wool based wound dressings was increased by $75 \%$, when wool fibres were grafted with antioxidant phenolic compounds by using tyrosinase. ${ }^{18}$ Tyrosinase catalyzed cross-linking of collagen was found to enhance properties important for application in tissue engineering or as implants. ${ }^{19,20}$ Here, we present a new application of tyrosinase based cross-linking in the field of targeted drug delivery systems.

Tyrosinase is a bifunctional enzyme, catalyzing the $o$-hydroxylation of monophenols and the subsequent oxidation of $o$-diphenols to $o$-quinones [EC 1.10.3.1]. $O$-quinones are highly reactive species which undergo non-enzymatic reactions with amino acid residues containing amino and sulfhydryl groups such as cysteine or lysine. The degree of cross-linking highly depends on the accessibility of target amino acids in the substrate protein. If the substrate is not accepted by the enzyme due to a very tight structure or due to its size, the use of low molecular weight compounds (e.g. natural phenolic molecules), can enable the enzymatic cross-linking as reported by Thalmann et al. ${ }^{21}$ The phenolic compounds are oxidized by tyrosinase and can further react with the amino and sulfhydryl groups of the protein backbones of larger and less accepted substrates, such as HSA and monoclonal antibodies (mAbs), present in solution. Here we tested six different phenolic compounds for their ability to work as an intermediate substrate to cross-link HSA and a mouse IgG mAb.
Tyrosinase based cross-linking could be a powerful alternative to this conventional strategy. This method not only shortens expenditure of time but also avoids the use of expensive and toxic cross-linking chemicals and reducing reagents, since no preparation of HSA and mAb is necessary prior to conjugate production.

\section{Results and discussion}

\section{Cross-linking}

The objective of this work was to investigate the ability of tyrosinase from Agaricus bisporus ( $A b \mathrm{~T}$ ) to cross-link mAb and HSA for the production of targeted drug delivery systems. No cross-linking products were found when either free mAb plus enzyme or free HSA plus enzyme were used, according to SDSPAGE analysis. Likewise, when the combination of HSA, mAb and enzyme was tested, no cross-linking product was found (Fig. 1). This could be due to limited accessibility of tyrosine residues within the native proteins. Previously, it has been reported that due to the opened structure, partially unfolded proteins were better substrates for $T$. reesei tyrosinase. ${ }^{22}$ However, in this study partial unfolding of HSA by using chaotropic agents or temperature did not lead to cross-linking products (data not shown). An alternative way to cross-link proteins using tyrosinase is the addition of natural low molecular weight phenolic compounds like caffeic acid or coumaric acid which are suitable substrates for tyrosinase. These phenolic molecules act as cross-linkers after enzymatic oxidation to the corresponding $o$-quinones. ${ }^{21} \mathrm{O}$-quinones can

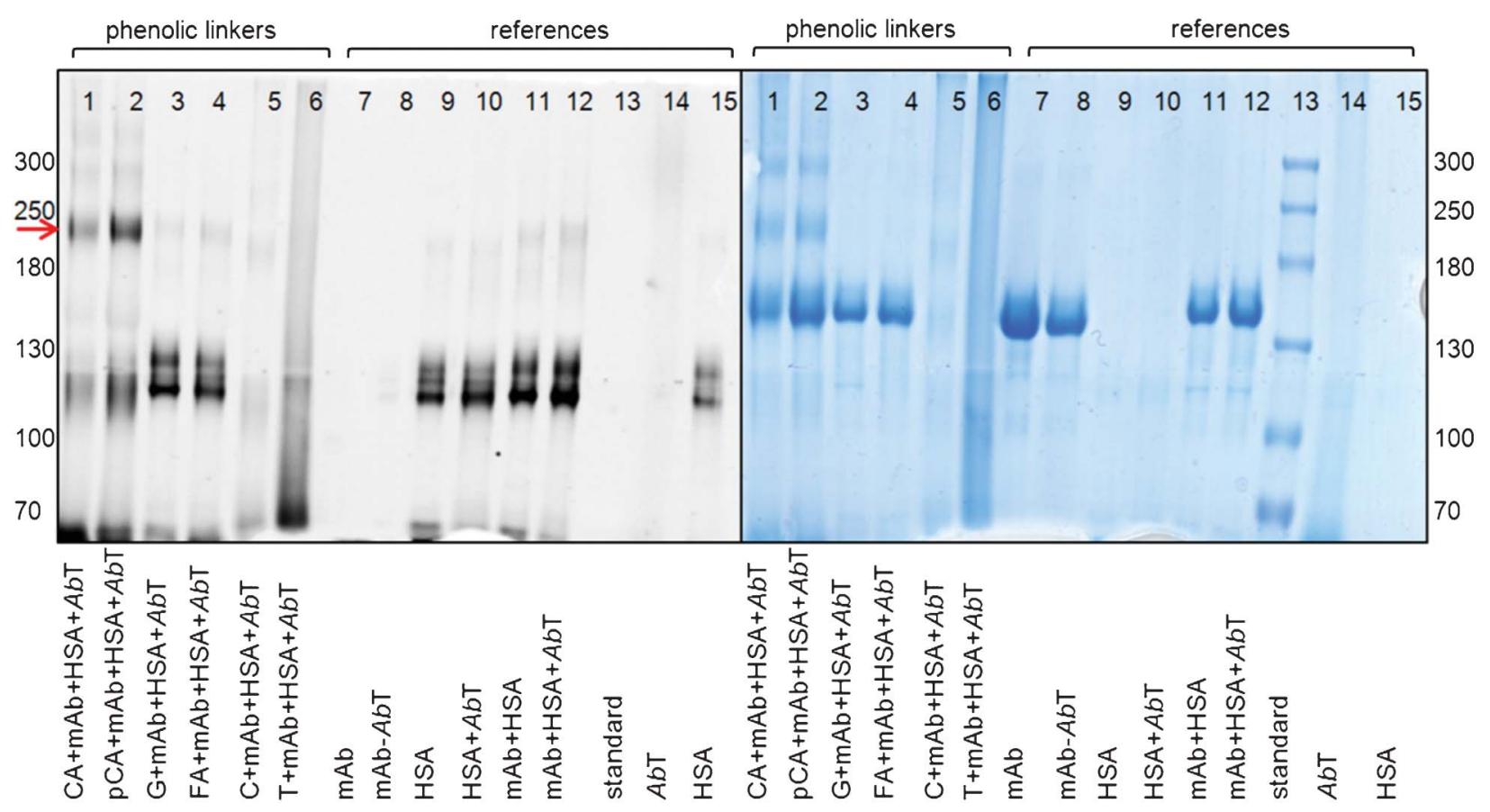

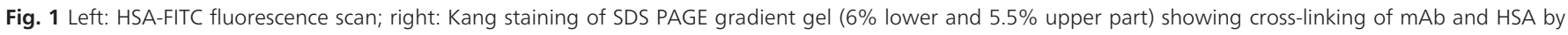

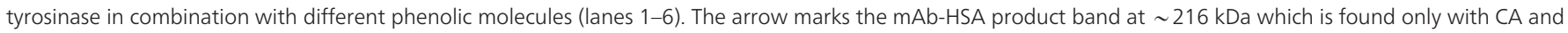
pCA (lane 1 and 2). Lanes 7-15 show references where HSA, phenolic molecules, mAb and AbT were replaced with reaction buffer. 
Table 1 LC-MS/MS analysis of mAb-HSA conjugates. HSA and mAb were cross-linked with tyrosinase in the presence of CA. The SDS-PAGE band at 216 kDa was subjected to tryptic digestion followed by LC-MS/MS analysis. The table shows the most abundant peptides found in a NCBI protein database search

\begin{tabular}{|c|c|c|c|c|c|c|}
\hline 1 & 148 & 24 & 49 & 69367 & Homo Sapiens & Serum albumin preprotein \\
\hline
\end{tabular}

undergo a spontaneous Michael-like addition with amino- and sulfhydryl groups of amino acid residues found in the backbone of proteins ${ }^{21,23}$ and thereby cross-link proteins.

We tested six different phenolic molecules (caffeic acid (CA), $p$-coumaric acid ( $p \mathrm{CA})$, guiacol $(\mathrm{G})$, ferulic acid (FA), catechol (C) and tyrosine (T)) for their ability to serve as cross-linkers between HSA and mAb. Indeed, new bands above the original $\mathrm{mAb}$ and HSA bands were seen in SDS-PA gels indicating cross-linking (Fig. 1). For analysis purposes HSA was labeled covalently with the fluorescent dye fluorescein 5(6)-isothiocyanate (FITC) prior to enzymatic coupling reactions. Bands in the molecular weight range of $\sim 216 \mathrm{kDa}$, visible both in the fluorescent scan, signaling the presence of HSA (which was covalently labeled with FITC for analysis) and after Kang staining can be assigned to mAb-HSA $1: 1$ conjugates. To verify the composition of the mAb-HSA conjugate, the band was cut out of the gel and subjected to tryptic digestion followed by LC-MS/MS analysis. Truly, HSA and mAb were identified as the most prominent hits in a complete NCBI protein database search of the conjugate digests peptide mass patterns (see Table 1). This clearly confirms the nature of the conjugate and excludes the possibility of artifacts potentially resulting from HSA oligomers. Analyzing further cross-linking products, the band at $\sim 300 \mathrm{kDa}$, which is only visible by Kang staining, can be attributed to mAb-mAb, while the band at $\sim 282 \mathrm{kDa}$, expectedly visible in fluorescent scans, matches the predicted molecular weight of HSA-mAb-HSA. Again, no bands representing HSA-HSA oligomers were detected.

Interestingly, mAb-HSA conjugates were only formed in the presence of CA and $p \mathrm{CA}$ although all 6 linker molecules are substrates for $A b \mathrm{~T}$ (data not shown). When comparing the chemical structures of these molecules, the common structural elements of CA and pCA are the free hydroxyl groups located in position 4 for $p$ CA and position 4 and 3 for CA, and the acrylic acid group in position 1. Also, FA has an acrylic acid group however, instead of a second hydroxyl group, a methoxy group is positioned next to the hydroxyl group which can lead to steric hindrance. ${ }^{23}$ Another reason for lack of activity with FA could be, that no further oligomerization is possible after a 5-5 dimerisation of FA, which is the preferred form of linkage due to lower heat of formation values. ${ }^{24}$ Looking at G, C and T, all three molecules do not contain acrylic acid moieties. It should be mentioned that in contrast to previous findings ${ }^{21}$ no difference was found between monophenols and biphenols. We assume that the structural elements, hydroxyl- and acryl groups are the key to a successful cross-linking of $\mathrm{mAb}$ and HSA. Further, in absence of mAb (HSA respectively), no defined new band in higher molecular weight ranges was seen (Fig. 2); however a smear in the range of 50-65 kDa (150$160 \mathrm{kDa}$ in the case of the reference not containing HSA) was

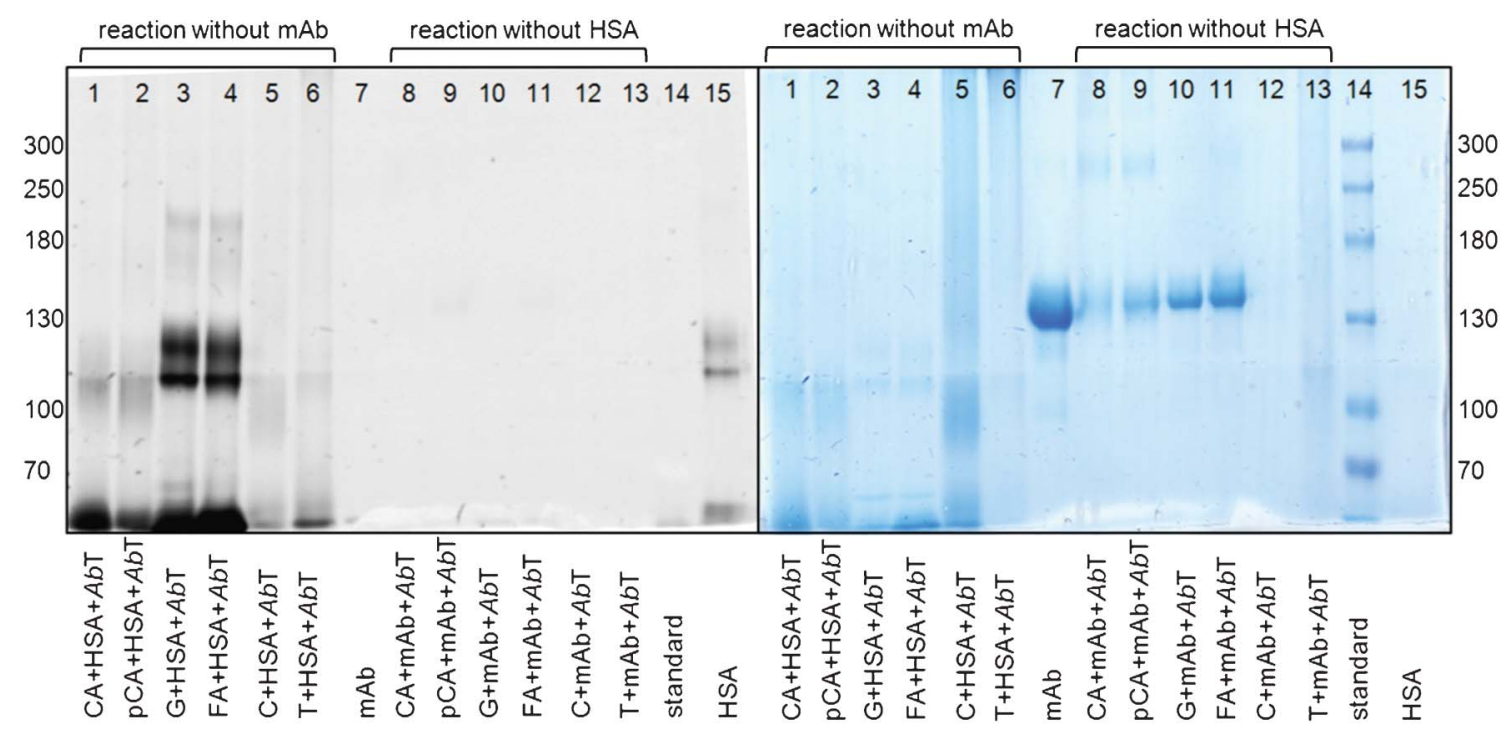

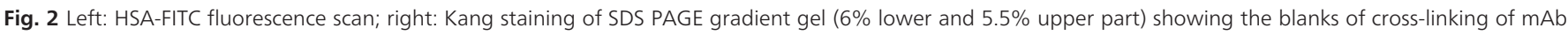

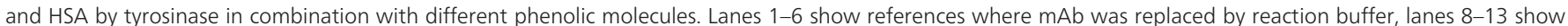
references where HSA was replaced by reaction buffer. 
visible (Fig. 2 lanes 1,2 and 8,9). This indicated that these phenolic molecules are not only acting as a single linker molecule but also oligomerize after oxidation by $A b \mathrm{~T}$. Higher molecular weight bands of HSA in presence of $\mathrm{CA} / p \mathrm{CA}$ and $A b \mathrm{~T}$ can be explained by this theory, which result from the attachment of different amounts of CA/ $p$ CA oligomers to the protein (Fig. 2). Oligomerisation of phenolics was also seen in size exclusion chromatography (SEC) (Fig. 3, elution volume of 6-7 mL). For T even larger products are visible which is most likely due to eumelanin production, since $\mathrm{T}$ is a natural substrate for tyrosinase which is converted to the polymer melanin during melanogenesis in insects, animal and plants. ${ }^{25}$ Only for CA and $p$ CA a different peak pattern in the elution volume range of $7-10 \mathrm{~mL}$ is visible which is correlated to the mAb-HSA conjugate.

A reaction mechanism for the enzymatic cross-linking of $\mathrm{mAb}$ and HSA is hypothesized (Fig. 4). In a first step, the phenolic compound is oxidized to the corresponding quinone. Subsequently, either mAb or HSA can bind through amino- or sulfhydryl groups. In the next step, the phenolic moiety is reoxidized and eventually further phenolic molecules attach. Finally, coupling of $\mathrm{mAb}-(\mathrm{CA})_{n}$ and $\mathrm{HSA}-(\mathrm{CA})_{m}$ lead to the corresponding conjugates.

\section{Binding characteristics of the enzymatically conjugated $\mathbf{m A b}$}

The mAb used in this study recognizes a common epitope on the beta-chain of human HLA-DR and HLA-DP. ${ }^{26}$ DR and DP are isotypes of the human MHC class II molecule, which is found on antigen-presenting cells like macrophages, dendritic cells or B cells. The MHC class II molecule consists of two polypeptide chains (alpha and beta), which are sub-structured into two subdomains respectively (alpha 1 and alpha 2, beta 1 and beta 2). A small antigenic peptide can be bound in the region between alpha 1 and beta 1 and be presented to CD4positive T cells. ${ }^{27}$

ELISA and flow cytometry analysis were performed to study the impact of enzymatic cross-linking and conjugation with HSA on the binding characteristics of the anti-MHC class II mAb. Both tests are based on the comparison of the binding pattern of the HSA-mAb conjugate and the free mAb to MHC class II molecules. Prior to ELISA and flow cytometry, the mAbHSA conjugate (cross-linked by CA) was purified using SEC and fractions of peak III were pooled (Fig. 5, inlet). First, the binding ability of the mAb-HSA conjugate was tested by ELISA. As shown in Fig. 5, the binding of the mAb-HSA conjugate to the MHC class II molecules in lysate of MHC II positive Raji B cells was similar to the binding of the free mAb. Presence of HSA in the conjugate was proved by detection with 1) LC-MS/ MS and 2) and anti-HSA antibody. The signal of the HSA-mAb was one third higher than when incubated with secondary antibody only showing the presence of HSA. In contrast, no signal enhancement was visible when only free mAb was used in the primary incubation. Further we conclude from this experiment, that no unspecific binding of reference samples, including free HSA, isotype antibody and blocking buffer, to MHC class II molecules is detectable.

Second, by flow cytometry analysis we tested the binding of the mAb-HSA conjugate to MHC class II molecules on living cells by employing MHC class II positive Raji B cells and MHC class II negative Jurkat $\mathrm{T}$ cells. Interestingly, the binding pattern of mAb-HSA was similar to the binding pattern of free $\mathrm{mAb}$ (Fig. 6) indicating that the HSA-conjugated $\mathrm{mAb}$ recognized its antigen well. However, the conjugates displayed also weak reactivity with the MHC class II negative cells. This might be due to the induction of a slight unspecificity upon AbT-mediated conjugation that might be controlled by optimization of the coupling conditions. Alternatively, the conjugation could also increase the affinity of the anti-MHC class II mAb revealing a weak expression of its epitope on Jurkat $\mathrm{T}$ cells.

\section{Experimental}

\section{Materials}

Tyrosinase from Agaricus bisporus ( $A b \mathrm{~T}$ ) was purchased from Sigma-Aldrich (Steinheim, Germany). The activity was measured with an adapted method from $\operatorname{Robb}^{28}$ and calculated to be $2.41 \mathrm{U} \mathrm{mL}^{-1}$ where one unit represents the amount of enzyme, which converts $1 \mu \mathrm{mol}$ of L-tyrosine to L-dopaquinone. Human serum albumin (HSA) and fluorescein 5(6)-isothiocyanate (FITC) were purchased from Sigma-Aldrich (Steinheim, Germany). Mouse monoclonal antibody (mAb) to HLA-DR+DP (MEM-136, IgG1), mouse IgG1 isotype control mAb (MOPC-21) and mouse mAb to human serum albumin (AL-01 (IgG1)) were a kind gift from EXBIO Praha, a.s. (Vestec, Czech Republic). Mouse mAb to alpha-fetoprotein that was used as an isotype control in flow cytometry experiments (AFP-12, IgG1) was a kind gift from Dr Václav Hořejší (Institute of Molecular Genetics, Academy of Sciences of the Czech Republic, Prague, Czech Republic). Allophycocyanin (APC)-conjugated goat anti-mouse IgG+IgM antibody was purchased from Jackson ImmunoResearch, West Grove, PA, USA and antimouse IgG-HRP conjugate from Amersham. ECL ${ }^{\mathrm{TM}}$ Western Blotting System was purchased from GE-Healthcare (Vienna, Austria). All other reagents used were of analytical grade.

\section{Methods}

Cross-linking reaction. For enzymatic cross-linking, stock solutions of all proteins were freshly prepared in $0.1 \mathrm{M}$ sodium phosphate buffer ( $\mathrm{pH}$ 6.8) in following concentrations: mAb $4.7 \mathrm{mg} \mathrm{mL}^{-1}$, HSA-FITC $10 \mathrm{mg} \mathrm{mL}^{-1}$ and $A b \mathrm{~T} 1.0 \mathrm{mg} \mathrm{mL}^{-1}$.

HSA was covalently labeled with FITC prior to the crosslinking experiment. Therefore $4 \mathrm{~mL}$ of a HSA solution $(10 \mathrm{mg}$ $\mathrm{mL}^{-1}$ in $50 \mathrm{mM}$ bicarbonate buffer, $\mathrm{pH}$ 9.0) were mixed with $200 \mu \mathrm{L}$ of FITC solution ( $5 \mathrm{mg} \mathrm{mL}^{-1}$ in DMSO). This mixture was shaken for four hours at $25{ }^{\circ} \mathrm{C}$ under light protection. Thereafter the mixture was purified by size exclusion chromatography using a HiPrep 26/10 Desalting column (GE Healthcare Europe GmbH, Vienna, Austria) installed on a Äkta Purifier system (Amersham Pharmacia Biotech, Uppsala, Sweden), with $50 \mathrm{mM}$ potassium phosphate and $100 \mathrm{mM}$ $\mathrm{NaCl}(\mathrm{pH} 7.4)$ as eluent, at the flow rate of $3 \mathrm{~mL} \mathrm{~min}^{-1}$ to remove excess FITC. Fractions showing absorbance at 280 and $485 \mathrm{~nm}$ were collected and reduced to a volume of $4 \mathrm{~mL}$ using a Vivaspin $30 \mathrm{kD}$ centrifugal separator at $5000 \mathrm{rpm}$ using Biofuge primo (Heraeus, Buckinghamshire, England). 

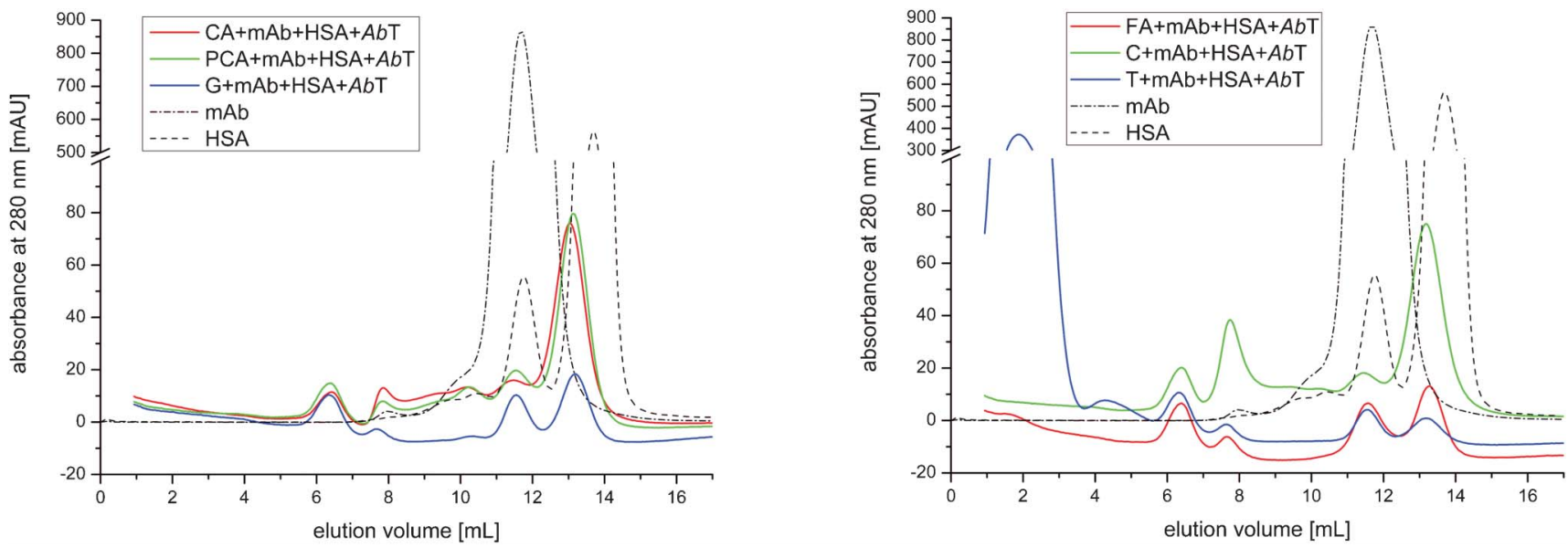

Fig. 3 SEC analysis of enzymatic cross-linking products of mAb and HSA cross-linked with AbT in the presence of different phenolic molecules (2 mM CA, $p C A$, G, FA, C and $1 \mathrm{mM} \mathrm{T}$ ).

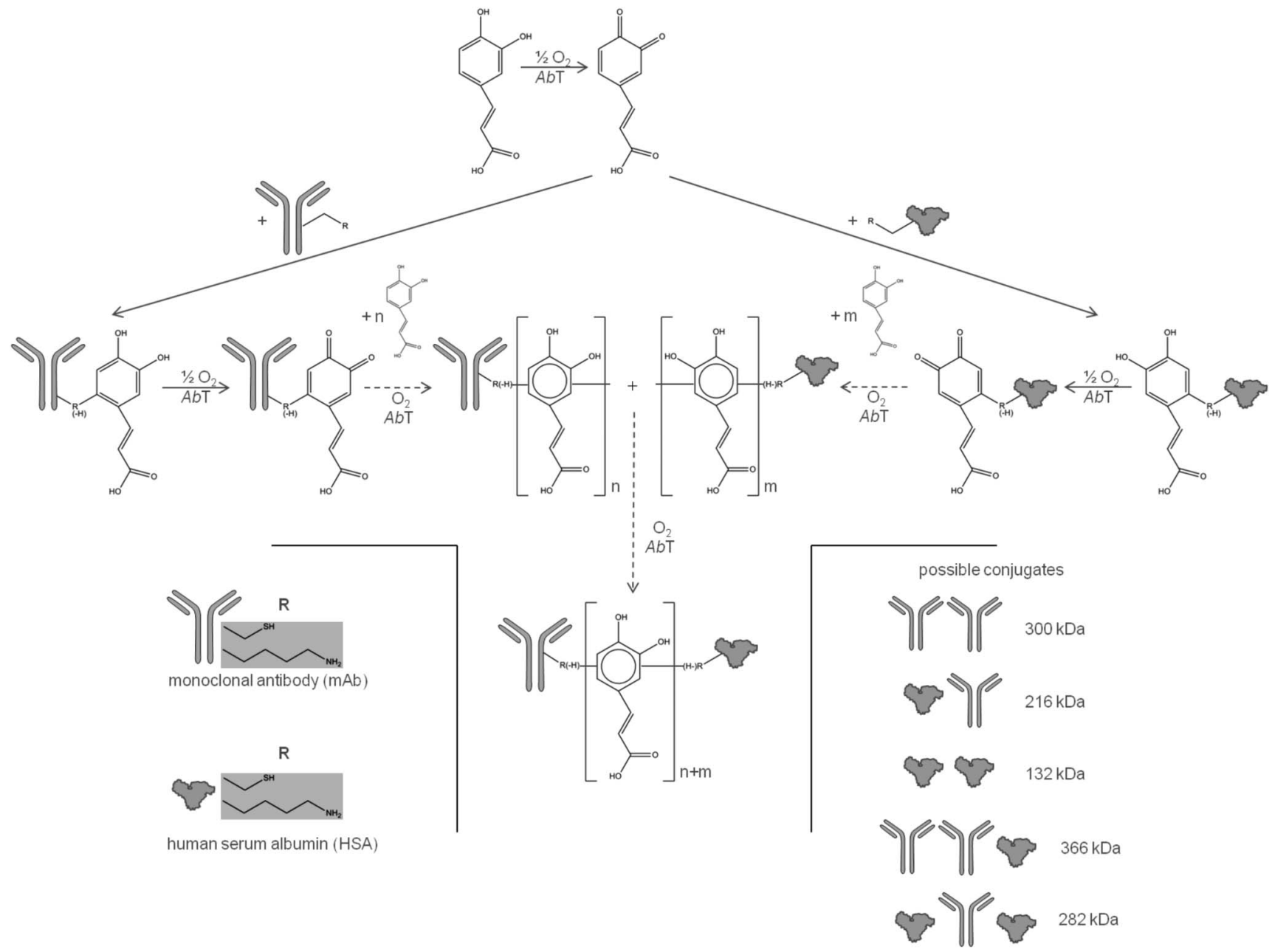

Fig. 4 Suggested reaction scheme for tyrosinase induced cross-linking of mAb and HSA. In a first step, phenolic cross-linkers (exemplary shown for CA) containing hydroxyl- and acrylic acid groups are oxidized by tyrosinase to give the corresponding reactive quinones. In the next step, these quinones couple to $-\mathrm{NH}_{2}$ or $-\mathrm{SH}$ residues of mAb and/or HSA. These adducts can either cross-link directly or after incorporation of further phenolic cross-linkers (as indicated by enlarged bands in SDS-PAGE analysis). Right: possible conjugates being generated during enzymatic cross-linking process of mAb and HSA, including predicted molecular weights. 


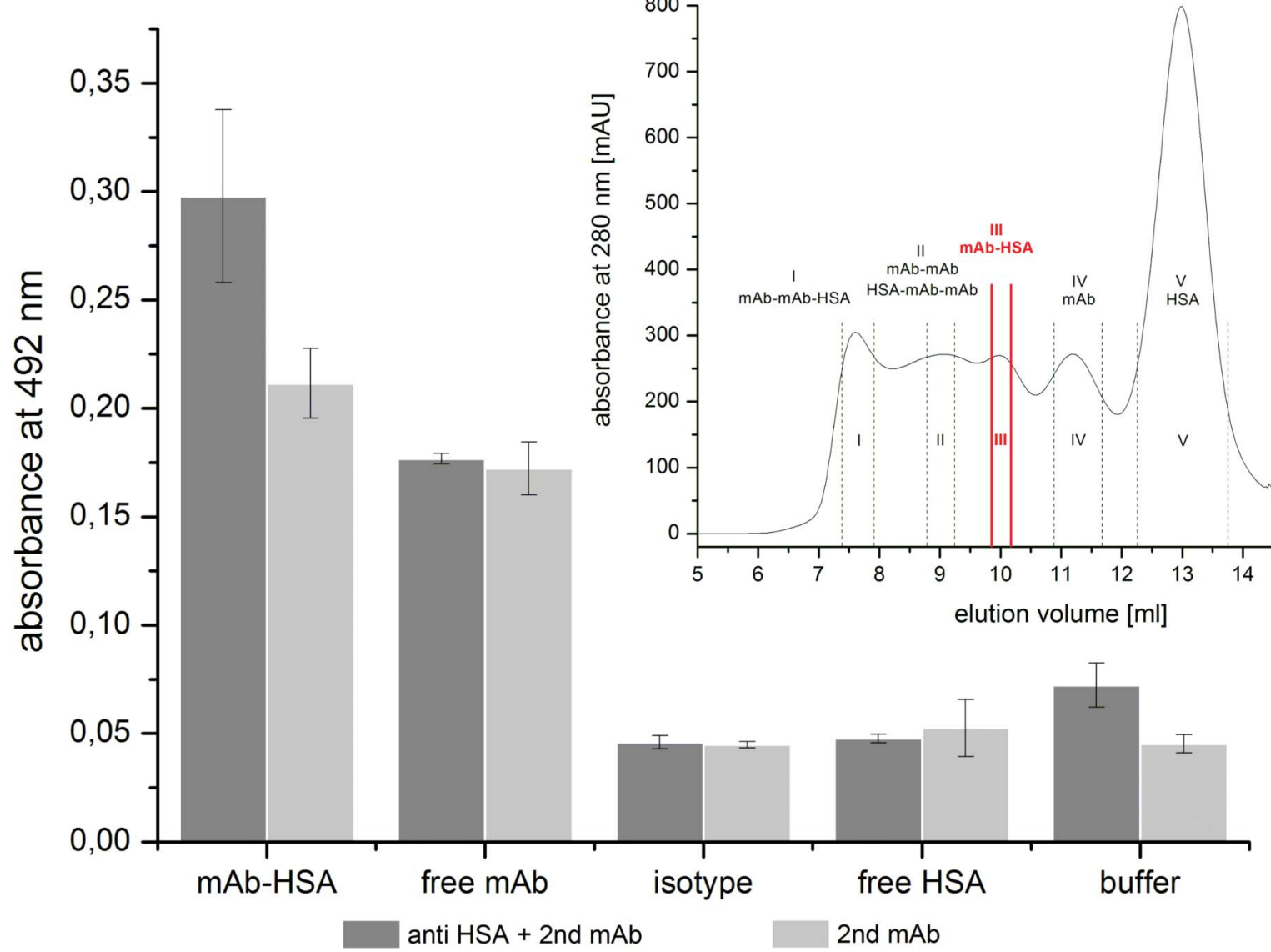

Fig. 5 ELISA to compare the binding properties of enzymatically cross-linked mAb-HSA conjugate. Lysates of MHC class II positive Raji B cells were analyzed with fraction III of SEC in comparison to free mAb, free HSA, isotype control mAb AFP-12 and blocking buffer alone. Binding was detected by incubation with an anti-HSA antibody followed by incubation with a horseradish peroxidase-labeled secondary antibody. Control incubation was performed with the secondary antibody only. Inlet: SEC of enzymatic mAb-HSA coupling using $2 \mathrm{mM}$ CA as phenolic linker molecule. Products were eluted using a low flow rate of $0.2 \mathrm{~mL} \mathrm{~min}^{-1}$, fractions of peak III were collected and concentrated.

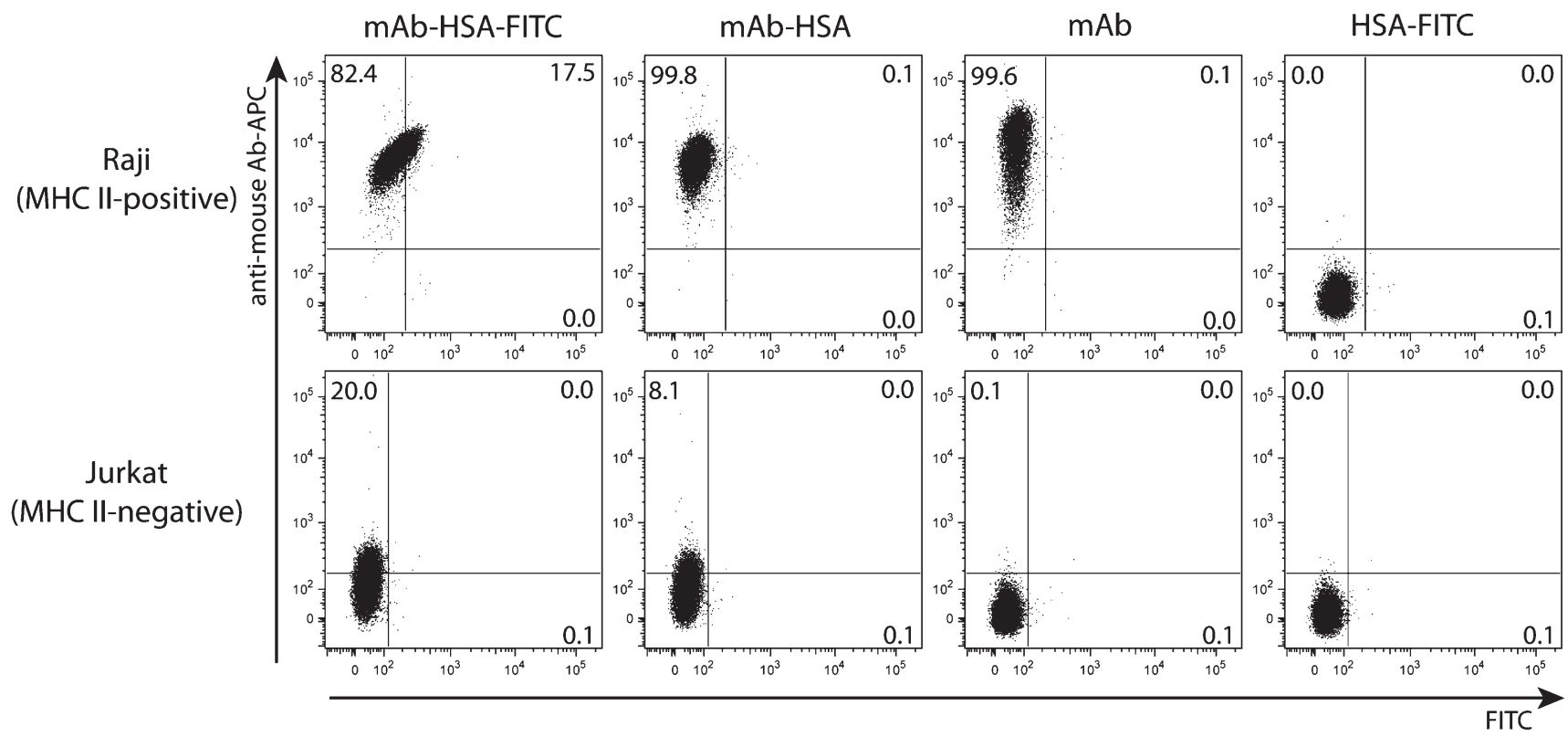

Fig. 6 Flow cytometry analysis of MHC class II positive Raji B cells and MHC class II negative Jurkat T cells to compare the binding properties of mAb-HSA conjugates (FITC-labeled and non-labeled), unconjugated FITC-labeled HSA and unconjugated anti-MHC class II mAb MEM-136. The binding was visualized using APC-labeled secondary antibody to mouse $\lg (y$ axis). The fluorescence intensity of FITC is shown on the $x$ axis of the two-dimensional dot plot diagrams. Numbers represent percentage of cells in the quadrants in comparison to the isotype control mAb AFP-12 that was set to a cut-off of $0.1 \%$. Images are representative of three independent experiments. 
Six different phenolic molecules (caffeic acid (CA), $p$-coumaric acid ( $p C A)$, guiacol (G), ferulic acid (FA), catechol (C) and tyrosine (T)) were tested for their linker ability. Therefore they were dissolved in $50 \% \mathrm{EtOH}$ in $\mathrm{H}_{2} \mathrm{O}$ to a concentration of 30 $\mathrm{mM}$ (tyrosine $2 \mathrm{mM}$ ).

For the cross-linking experiment mAb $\left(\begin{array}{lll}6.0 & \mu \mathrm{M}\end{array}\right)$ was combined with the double molar amount of HSA $(12.0 \mu \mathrm{M})$ and $2 \mathrm{mM}$ of each phenolic linker molecule $(1 \mathrm{mM}$ for tyrosine) in a total reaction volume of $100 \mu \mathrm{L}$. The reaction was started by adding $A b \mathrm{~T}$ in the dosage of $0.55 \mathrm{U} \mathrm{mL}^{-1}$. For all cross-linking experiments reference samples were prepared by replacing HSA, phenolic molecules, mAb, and $A b \mathrm{~T}$, respectively with reaction buffer. All samples were shaken at $450 \mathrm{rpm}$ at a temperature of $25{ }^{\circ} \mathrm{C}$ for $24 \mathrm{~h}$.

\section{Analysis}

Size exclusion chromatography (SEC). For product analysis SEC was performed using a Superdex 200 10/300 GL (GE Healthcare Europe $\mathrm{GmbH}$, Vienna, Austria) installed on an Äkta Purifier system (Amersham Pharmacia Biotech, Uppsala, Sweden). 0.1 M NaPh buffer ( $\mathrm{pH}$ 6.8) was used as eluent, at a flow rate of $0.4 \mathrm{~mL} \mathrm{~min}^{-1}$, and absorbance was detected at 280 nm.

For product purification the centrifuged crude mixture was desalted using a $5 \mathrm{~mL}$ HiTrap $^{\mathrm{TM}}$ column (GE Healthcare Europe $\mathrm{GmbH}$, Vienna, Austria) at a flow rate of $1 \mathrm{~mL} \mathrm{~min}{ }^{-1}$, followed by SEC using a Superdex 200 10/300 GL with a lower

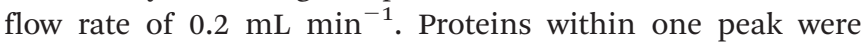
pooled and concentrated using a Vivaspin 2 .

Sodium dodecyl sulfate polyacrylamide gel electrophoresis (SDS PAGE). SDS PAGE was performed using a Tris-HCl gradient gel (6\% lower-5.5\% upper part). For molecular weight determination a high range protein standard (Spectra Multicolor High Range Protein Ladder, Fermentas) was used. The proteins were visualized with Coomassie Blue staining according to Kang. ${ }^{29}$ FITC labeled HSA was detected by fluorescent scanning (Ex. $488 \mathrm{~nm}$, Em. $530 \mathrm{~nm}$ ) of gels on a molecular imager equipped with Laser Scanner FXPro and analysed by Quantity One software (Bio-Rad).

Liquid chromatography-mass spectrometry (LC-MS/MS) analysis. Protein identification and internal sequence information was received from LC-MS/MS. The protein product band stained with Coomassie Brillant Blue R-250 was excised from the SDS gel and reduced, alkylated and digested with Promega modified trypsin according to the method of Shevchenko et al. ${ }^{30}$

Digests were separated by nano-HPLC (Agilent 1200 system, Vienna, Austria) equipped with a Zorbax 300SB-C18 enrichment column $(5 \mu \mathrm{m}, 5 \times 0.3 \mathrm{~mm})$ and a Zorbax 300SB-C18 nanocolumn $(3.5 \mu \mathrm{m}, 150 \times 0.075 \mathrm{~mm}) .40 \mu \mathrm{L}$ of sample was injected and concentrated on the enrichment column for 6 min using $0.1 \%$ formic acid as isocratic solvent at a flow rate of $20 \mu \mathrm{L} \mathrm{min}{ }^{-1}$. The column was then switched in the nanoflow circuit, and the sample was loaded on the nanocolumn at a flow rate of $300 \mathrm{~nL} \mathrm{~min}^{-1}$. Separation was carried out using the following gradient, where solvent $\mathrm{A}$ is $0.3 \%$ formic acid in water and solvent $\mathrm{B}$ is a mixture of acetonitrile and water ( $4: 1$, by vol.) containing $0.3 \%$ formic acid: 0-6 min: 13\% B; 6-35 $\min : 13-28 \% \mathrm{~B} ; 35-47 \mathrm{~min}: 28-$ 50\% B, 47-48 min: 50-100\% B; 48-58 min: 100\% B; 58-59 min: $100-13 \%$ B; 59-70 min: re-equilibration at 13\% B. The sample was ionized in the nanospray source equipped with nanospray tips (PicoTipTM Stock\# FS360-75-15-D-20, Coating: 1P-4P, $15 \pm$ $1 \mu \mathrm{m}$ Emitter, New Objective, Woburn, MA, USA). It was analysed in a Thermo LTQ-FT mass spectrometer (Thermo Fisher Scientific, Waltham, MA, USA) operated in positive ion mode, applying alternating full scan MS ( $\mathrm{m} / \mathrm{z} 400$ to 2000) in the ion cyclotron and MS/MS by collision induced dissociation of the 5 most intense peaks in the ion trap with dynamic exclusion enabled.

The LC-MS/MS data were analysed by searching the NCBI public database downloaded on Aug 23rd, 2011 with Spectrum mill Rev. A.03.03.084 SR4 (Agilent, Vienna, Austria). Detailed search criteria for Spectrum Mill were: trypsin; max. missed cleavage sites: 2; carbamidomethylation at cysteine as fixed modification; variable modification: oxidised methionine; precursor mass tolerance $\pm 0.05 \mathrm{Da}$; product mass tolerance \pm 0.7 Da. Protein hits were subjected to automatic validation by Spectrum mill: for precursor charge of 2: score threshold 6.0, percent scored peak intensity (\%SPI) threshold 60.0, FwdRev score threshold 2.0 and rank 1-2 score threshold 2.0; for precursor charge of 1 : score threshold 6.0, \%SPI threshold 70.0, Fwd-Rev score threshold 2.0 and rank 1-2 score threshold 2.0; for precursor charge of 3: score threshold 8.0, \%SPI threshold 70.0, Fwd-Rev score threshold 2.0 and rank 1-2 score threshold 2.0.

\section{Antibody function}

Cells. The Jurkat T cell line E6.1 and the B cell line Raji were purchased from the American Type Culture Collection. Cells were maintained in RPMI 1640 medium supplemented with 2 $\mathrm{mM}$ L-glutamine, $50 \mu \mathrm{g} \mathrm{mL} \mathrm{m}^{-1}$ streptomycin, and $50 \mathrm{U} \mathrm{mL}^{-1}$ penicillin and $10 \%$ heat inactivated FCS (all from Invitrogen) in a humidified atmosphere with $5 \% \mathrm{CO}_{2}$ at $37{ }^{\circ} \mathrm{C}$.

Enzyme-linked immunosorbent assay (ELISA). To compare the binding of the mAb-HSA conjugate to that of the free $\mathrm{mAb}$ an ELISA was performed. Wells of ELISA plates (Greiner high and medium binding 96 well plates, Sigma-Aldrich, Steinheim, Germany) were coated with $50 \mu \mathrm{L}$ of Raji cell lysate $\left(1 \times 10^{5}\right.$ cells $\mathrm{mL}^{-1}$ ) for $12 \mathrm{~h}$. Cell lysate was obtained by centrifuging $10 \times 10^{6}$ cells at $350 \mathrm{~g}$, whereas the supernatant was discarded, and the cell pellet resolved in $100 \mu \mathrm{L}$ lysis buffer containing $20 \mathrm{mM} \mathrm{Na} \mathrm{HPO}_{4}, 1 \%$ detergent TritonX100 and $1 \mathrm{x}$ protease inhibitor cocktail (Roche) at $\mathrm{pH}$ 7.4. After washing with washing buffer $(0.05 \%$ detergent Tween 20 in PBS, pH 7.4) and blocking (1\% skimmed dry milk in washing buffer) the mAb-HSA conjugate and control samples (buffer, free mAb, AFP12 isotype control mAb, free HSA) were incubated for $1 \mathrm{~h}$ at $23{ }^{\circ} \mathrm{C}$. After washing, the samples were incubated for $1 \mathrm{~h}$ with an anti-HSA antibody followed by incubation with a horseradish peroxidase-labeled secondary antibody. A control incubation was performed where the samples were incubated with the secondary antibody only. The immune-reaction was visualized using $o$-phenylenediamine as a substrate (SigmaAldrich). The reaction was stopped by the addition of $3 \mathrm{M} \mathrm{HCl}$ and optical density was read in a microplate reader at $492 \mathrm{~nm}$. 
Flow cytometry. Cells were washed with staining buffer (PBS containing $1 \% \mathrm{BSA}$ and $0.02 \% \mathrm{NaN}_{3}$ ) and incubated for $30 \mathrm{~min}$ with $4.8 \mu \mathrm{g} \mathrm{mL} \mathrm{m}^{-1}$ human IgG on ice to prevent nonspecific binding of the mAbs to Fc receptors. Then, the FITC-labeled mAb-HSA conjugate, the non-labeled one, the free primary $\mathrm{mAb}$, the free FITC-conjugated HSA or the isotype control mAb AFP-12 (all $10 \mu \mathrm{g} \mathrm{mL} \mathrm{m}^{-1}$ ) was added and the cells were incubated for $30 \mathrm{~min}$ on ice. Cells were washed with staining buffer and incubated with APC-conjugated secondary antibody for $30 \mathrm{~min}$ on ice. After a final wash, cells were analyzed on an LSRII flow cytometer (BD Biosciences) and the data further processed with the FloJo software (Treestar). The live cells were gated according to their forward- and side-scatter characteristics and exclusion of dead cells using DAPI.

\section{Conclusion}

The enzymatic synthesis of mAb-HSA conjugates suitable for targeted drug delivery devices was investigated. We were able to demonstrate that the conjugated $\mathrm{mAb}$ is still recognizing its antigen with immunological tests comparing the binding ability of free mAb and the mAb-HSA conjugate to MHC class II molecules. The enzyme tyrosinase from Agaricus bisporus was used to cross-link HSA and mAb in the presence of phenolic molecules. Out of six phenolic compounds tested, CA and $p$ CA were able to act as a spacer and cross-linker. We assume that the acrylic acid moiety in combination with a mono- or biphenol is the crucial point for a successful crosslinking reaction. Conjugates were analyzed using SDS-PAGE and SEC. LC-MS/MS analysis of the peptide fragments, obtained by a tryptic digest of a product band at the molecular weight range of $\sim 216 \mathrm{kDa}$, which is the predicted molecular weight for a mAb-HSA conjugate, confirmed that this product consist of mAb and HSA. Consequently this one-step enzymatic cross-linking strategy not only represents a green alternative for conventional laborious methods but also avoids expensive and harmful chemical cross-linkers.

\section{Acknowledgements}

This work has received funding from the European Union Seventh Framework Programme (FP7/2007-2013) under grant agreement NMP4-LA-2009-228827 NANOFOL and FWF, DK: Metabolic and Cardiovascular Disease: W1226-B18. We thank Tamara Reiter, Graz University of Technology for technical support with SEC; Exbio from the Czech Republic for providing the mAbs and Britta Obrist, Medical University of Graz and the Austrian Centre of Industrial Biotechnology, for technical assistance with LC-MS/MS analysis.

\section{References}

1 A. Sparreboom, M. J. A. de Jonge and J. Verweij, Eur. J. Cancer, 2002, 38, 18-22.
2 N. Desilet, T. N. Campbell and F. Y. M. Choy, Curr. Issues Mol. Biol., 2010, 12, 143-146.

3 F. Fay and C. J. Scott, Immunotherapy, 2011, 3, 381-394.

4 S. C. Alley, N. M. Okeley and P. D. Senter, Curr. Opin. Chem. Biol., 2010, 14, 529-537.

5 U. Iyer and V. J. Kadambi, J. Pharmacol. Toxicol. Methods, 2011, 64, 207-212.

6 F. Kratz, J. Controlled Release, 2008, 132, 171-183.

7 A. Rollett, T. Reiter, P. Nogueira, M. Cardinale, A. Loureiro, A. Gomes, A. Cavaco-Paulo, A. Moreira, A. M. Carmo and G. M. Guebitz, Int. J. Pharm., 2012, 427, 460-466.

8 N. Endo, Y. Kato, Y. Takeda, M. Saito, N. Umemoto, K. Kishida and T. Hara, Cancer Research, 1987, 47, 1076-1080.

9 M. G. Anhorn, S. Wagner, J. Kreuter, K. Langer and H. von Briesen, Bioconjugate Chem., 2008, 19, 2321-2331.

10 I. Steinhauser, B. Spänkuch, K. Strebhardt and K. Langer, Biomaterials, 2006, 27, 4975-4983.

11 F. Hollmann, I. W. C. E. Arends, K. Buehler, A. Schallmey and B. Buhler, Green Chem., 2011, 13, 226-265.

12 F. Hollmann, I. W. C. E. Arends and D. Holtmann, Green Chem., 2011, 13, 2285-2314.

13 E. Yara-Varon, J. Eras Joli, M. Balcells, M. Torres and R. Canela-Garayoa, RSC Adv., 2012, 2, 9230-9236.

14 E. Selinheimo, K. Autio, K. Krijus and J. Buchert, J. Agric. Food Chem., 2007, 55, 6357-6365.

15 T. Chen, D. E. Heather, L.-Q. Wu and F. P. Gregory, Biopolymers, 2002, 64, 292-302.

16 A. Anghileri, R. Lantto, K. Kruus, C. Arosio and G. Freddi, J. Biotechnol., 2007, 127, 508-519.

17 T. Chen, R. Vazquez-Duhalt, C. Wu, W. E. Bentley and G. F. Payne, Biomacromolecules, 2001, 2, 456-462.

18 S. Jus, V. Kokol and G. M. Guebitz, Enzyme Microb. Technol., 2008, 42, 535-542.

19 S. Jus, I. Stachel, W. Schloegl, M. Pretzler, W. Friess, M. Meyer, R. Birner-Gruenberger and G. M. Guebitz, Mater. Sci. Eng., C, 2011, 31, 1068-1077.

20 L. S. Moreira Teixeira, J. Feijen, C. A. van Blitterswijk, P. J. Dijkstra and M. Karperien, Biomaterials, 2012, 33, 1281-1290.

21 C. R. Thalmann and T. Lotzbeyer, Eur. Food Res. Technol., 2002, 214, 276-281.

22 M. Hellman, M. Mattinen, B. Fu, J. Buchert and P. Permi, J. Biotechnol., 2011, 151, 143-150.

23 E. Selinheimo, D. NiEidhin, C. Steffensen, J. Nielsen, A. Lomascolo, S. Halaouli, E. Record, D. O'Beirne, J. Buchert and K. Kruus, J. Biotechnol., 2007, 130, 471-480.

24 L. E. B. Marinez and P. J. S. Valencia, Revista de la Sociedad Colombiana de Física, 2005, 37, 106-109.

25 S. Seo, V. K. Sharma and N. Sharma, J. Agric. Food Chem., 2003, 51, 2837-2853.

26 C. Koch, G. Staffler, R. Hüttinger, I. Hilgert, E. Prager, J. Černý, P. Steinlein, O. Majdic, V. Hořejší and H. Stockinger, Int. Immunol., 1999, 11, 777-786.

27 C. A. J. Janeway, P. Travers, M. Walport and M. J. Shlomchik, Immunobiology, 5th edition, The immune system in health and disease, Garland Science, New York, 2001.

28 D. A. Robb, in Copper proteins and copper enzymes, ed. R. Lontie, CRC Press, Florida, 1984, 207-240.

29 D. Kang, Y. S. Gho, M. Suh and C. Kang, Bull. Korean Chem. Soc., 2002, 23, 1511-1512.

30 A. Shevchenko, M. Wilm, O. Vorm and M. Mann, Anal. Chem., 1996, 68, 850-858. 